\title{
Full Mouth Rehabilitation a Multidisciplinary Approach: Case Report
}

\author{
Dr. Avantika Singh, Dr. Rohan Bhede, Dr. Manoj Chandak, Dr. Ajay Saxena
}

\begin{abstract}
A desire to look attractive is no longer taken as a sign of vanity. Since the face is the most exposed part of the body, and the mouth a prominent feature, teeth are getting a greater share of attention. The severe wear of anterior teeth facilitates the loss of anterior guidance, which protects the posterior teeth from wear during excursive movement. The collapse of posterior teeth also results in the loss of normal occlusal plane and the reduction of the vertical dimension. This case report describes 77-year-old female, who had the loss of anterior guidance, the severe wear of dentition, and the reduction of the vertical dimension. Occlusal overlay splint was used after the decision of increasing vertical dimension by anatomical landmark, facial and physiologic measurement. Once the compatibility of the new vertical dimension had been confirmed, interim fixed restoration and the permanent reconstruction was initiated.
\end{abstract}

Keywords: Tooth wear, Vertical dimension of occlusion, Occlusal overlay splint

\section{Introduction}

The gradual wear of the occlusal surfaces of teeth is a normal process during the lifetime of a patient. However, excessive occlusal wear can result in pulpal pathology, occlusal disharmony, impaired function, and esthetic disfigurement.(1) Tooth wear can be classified as attrition, abrasion, and erosion depending on its cause.

A differential diagnosis is not always possible because, in many situations, there exists a combination of these processes(2). Therefore, it is important to identify the factors that contribute to excessive wear and to evaluate alteration of the VDO caused by the worn dentition(3).

In many cases, the vertical dimension of occlusion (VDO) is maintained by tooth eruption and alveolar bone growth. As teeth are worn, the alveolar bone undergoes an adaptive process and compensates for the loss of tooth structure to maintain the VDO. Therefore, VDO should be conservative and should not be changed without careful approach. $(4,5)$ Especially, increasing the VDO in bruxers puts a severe overload on the teeth and often results in the destruction of the restorations or teeth themselves.(4)

Therefore in such cases not only replacement of the lost tooth structure but also restoring the lost vertical dimensions besides imparting both the esthetics and function. Full mouth reconstruction is basically a set of procedures that are aimed at correcting an improper bite position as well as restoring chipped or worn out teeth.

\section{Case report}

A 77-year-old woman was referred for the treatment of her severely worn dentition. Her chief complaint was that she could not eat anything because her teeth were worn too much. Intraoral examination revealed a generalized loss of dental substance that was greater in the maxillary left incisors and the mandibular right incisors. Missing 26323646 was seen. The anterior teeth had sharp enamel edges, dentinal craters, and attritional wear. The facial type of patient was square and her lip seemed to be under strong tension. The patient did not have temporomandibular disorder history and soreness of the mastication muscles, but the discrepancy between centric occlusion (CO) and maximum intercuspal position (MIP) was found when she was guided to $\mathrm{CR}$ with bimanual technique.

To determine whether VDO had been altered, the following aspects were investigated $(1,5,11)$

1. History of wear: Physiologic wear can be compensated by tooth eruption in general, but the accelerated wear may exceed the rate of eruption. The patient liked vegetables and acidic fruits. Her favorite food was tough and fibrous. In addition, maxillary posterior base metal prostheses might accelerate the wear of mandibular acrylic resin teeth and unbalance of wear rate.

2. Phonetic evaluation: If the distance between the incisal edge of the mandibular incisors and lingual surface of the maxillary incisors is about $1 \mathrm{~mm}$, it makes normal $/ \mathrm{s} /$ sound. The patient's increased space altered $/ \mathrm{s} /$ sound to $/ \mathrm{J} /$.

3. Interocclusal rest space: The patient's interocclusal rest space that was measured between nose tip and chin tip was $5-6 \mathrm{~mm}$ that was greater than the normal value, $2-4 \mathrm{~mm}$.

4. Facial appearance: Wrinkles and drooping commissures around mouth were observed.

The possible causes of patient's worn dentition that might include posterior interferences, parafunctional eating habit, and dental ignorance were explained to the patient. And the options of treatment 
plan were restoring mandibular edentulous posterior region with implants or removable partial denture, full mouth rehabilitation with metal ceramic restoration with or without crown lengthening procedure. The patient was scared of implant surgery, so the option of implant installation was excluded. Also the patient did not want to get multiple crown lengthening procedures and endodontic treatments to restore the worn teeth in the insufficient space. As there was clinical evaluation of reduced VDO, full mouth rehabilitation with increasing VDO was planned.

Before starting with the prosthetic management intentional root canal treatment was done with 1117 21222331333435414243 followed by permanent restoration.

Under local anesthesia $2 \%$ lidocain ,adrenaline 1:20,000 was administered, conventional root canal procedure was carried out under all aseptic conditions. Endodontic therapy was completed over 2 weeks under multiple sittings.

The patient's casts were mounted on a semi-adjustable articulator (Hanau ${ }^{\mathrm{TM}}$ Modular Articulator; Whip Mix Corp., Louisville, USA) using a face-bow record and an interocclusal record that was made with the aid of a Lucia jig and polyvinylsiloxane occlusal registration material (EXABITE II; GC Corp., Tokyo, Japan). The new VDO was set by $5 \mathrm{~mm}$ increase in the incisal guidance pin of the articulator. Because the patient's interocclusal rest space was $2-3 \mathrm{~mm}$ larger on the premolar area than normal distance, the actual increase were determined $3 \mathrm{~mm}$ in the anterior teeth and $1-2 \mathrm{~mm}$ in the posterior teeth. The splint was designed to offer bilateral contacts of all posterior teeth in centric relation and guides of the anterior teeth in excursive movement. The anterior guidance disoccluded the posterior teeth in all jaw position except centric relation.

The adaptation of patient to the increased VDO was evaluated during 1-month trial period. No muscle tenderness and temporomandibular discomfort was found. The method of increasing VDO with the splint was used to determine desirable VDO of the fixed interim prostheses. After taking CR record using Lucia jig and wax-rim, diagnostic wax-up was performed . Autopolymerizing acrylic resin (ALIKETM; GC America, ALSIP, USA) provisional crowns were fabricated using a vacuum formed matrix (Drufolen H; DreveDentamid GmbH, Unna, Germany) that was produced from the diagnostic wax-up, and mandibular provisional RPD was made to fit provisional crowns. The provisional fixed restorations were cemented with temporary cement (FREEGENOL TEMPORARY PACK; GC Corp., Tokyo, Japan), and the patient's adaptation was monitored.

For three months, interim restorations were adjusted, and used as a guide for the definitive oral rehabilitation. During this period, the patient's condition and functions, such as muscle tenderness, discomfort of TMJ, mastication, range of the mandibular movements, swallowing, and speech, were evaluated. Improvement in mastication, speech, and facial esthetics confirmed the patient's tolerance to the new mandibular position with the restored VDO. The anterior guidance and posterior disocclusion on excursive movement were established.

Adjusted occlusion was transferred to customized anterior guide table, which was made with acrylic resin (PATTERN RESIN; GC Corp, Tokyo, Japan).

Final preparation was performed, and definitive impressions were made with polyvinylsiloxane impression material (Extrude; Kerr Corp., Romulus, Germany). Bite registration was taken using provisional crown and occlusal registration material (StoneBite; DreveDentamid GmbH, Unna, Germany) by half and half .

Porcelain fused to metal restorations were made using customized anterior guide table and cemented with resin modified glass ionomer cement (FujiCEM; GC America, Alsip, USA). Because the patient's anterior guidance table was used in the production of definitie restoration, the amount of occlusal adjustment on the lingual surface of maxillary anterior teeth was minimal. Individual tray with polyvinylsiloxane (EXAMIXFINE; GC Corp., Tokyo, Japan) was used for the impression of mandibular RPD. The impression on posterior alveolar ridge was taken once more with the individual tray which is attached to the RPD framework, and the altered cast was made. After the adaptation of RPD framework and the trial of wax denture were done, the definitive mandibular RPD was fabricated and delivered with minor occlusal adjustment. The prostheses were designed using mutually protected occlusion. The anterior teeth protected the posterior teeth from excursive force and wear, and posterior teeth supported the bite force. Oral hygiene instruction and regular check-up were administered.

\section{Discussion}

In 1984, Turner (1) classified the treatment of a severely worn dentition by the amount of the loss of VDO and available space to restore. The etiology of tooth wear is multifactorial, and clinical controlled trials of restorative and prosthodontic approaches are limited in quantity and quality. In addition, lack of evidence regarding the long-term outcomes of treatment methods and materials cause difficulty in clinical decisionmaking(6)Nonetheless, the composite resin restoration could not be used for the patient in this case. The remaining tooth structures were too small to have sufficient retention of composite resin, and the surveyed crowns to support RPD were necessary. Therefore, the conventional treatment modality that includes a trial overlay splint, provisional restoration, careful monitoring, and definitive prosthesis, was chosen. 
In previous literature, the wearing time of overlay splint and provisional crown is various. The trial period of overlay prostheses which are reversible and conservative is between 3 weeks and 5 months, and that of intensive fixed provisional prostheses is $2-6$ months. $(1,5,7,8,9)$. In this case, the patient was carefully monitored for 1 month to evaluate the adaptation to the removable occlusal overlay splints. (10)Also the patient's adaptation to the provisional restoration was monitored for 3 months(11)The trial period is relatively shorter than the other case report, but discomfort, wear, and muscle fatigue were not observed during that period. The increase of VDO was determined not by standardized esthetic golden proportion of anterior teeth but by patient's physiologic factor like interocclusal rest space and speech. If the increase of VDO was decided arbitrarily without close evaluation, multiple complications would happen and longer treatment period might be needed. Depending on the patient's situation and adaptation ability, the interim period can be modified, and the careful evaluation and monitoring may shorten the overall treatment duration.

The education on wearing RPD is necessary. Regular check-up for the occlusal adjustment and RPD fitting is essential.

\section{Conclusion}

In this clinical report, raising vertical dimension of occlusion using removable occlusal overlay splint and following fixed provisional based on accurate diagnosis showed successful full mouth rehabilitation for severely worn down dentition.

\section{References}

[1]. Turner KA, Missirlian DM. Restoration of the extremely worn dentition. J Prosthet Dent. 1984; 52:467-474.

[2]. Smith BG. Toothwear: aetiology and diagnosis. Dent Update. 1989;16:204-212.

[3]. Prasad S, Kuracina J, Monaco EA., Jr Altering occlusal vertical dimension provisionally with base metal onlays: a clinical report. J Prosthet Dent. 2008; 100:338-342.

[4]. Dawson PE. Functional Occlusion - From TMJ to smile design. 1st ed. New York: Elsevier Inc.; 2008. pp. $430-452$.

[5]. Jahangiri L, Jang S. Onlay partial denture technique for assessment of adequate occlusal vertical dimension: a clinical report. J Prosthet Dent. 2002; 87:1-4.

[6]. Johansson A, Johansson AK, Omar R, Carlsson GE. Rehabilitation of the worn dentition. J Oral Rehabil. 2008;35:548-566.

[7]. Hemmings KW, Darbar UR, Vaughan S. Tooth wear treated with direct composite restorations at an increased vertical dimension: results at 30 months. J Prosthet Dent. 2000;83:287-293.

[8]. Sato S, Hotta TH, Pedrazzi V. Removable occlusal overlay splint in the management of tooth wear: a clinical report. J Prosthet Dent. 2000; 83:392-395.

[9]. Brown KE. Reconstruction considerations for severe dental attrition. J Prosthet Dent.1980;44:384-388.

[10]. Ganddini MR, Al-Mardini M, Graser GN, Almog D. Maxillary and mandibular overlay removable partial dentures for the restoration of worn teeth. J Prosthet Dent. 2004;91:210-214.

[11]. Yunus N, Abdullah H, Hanapiah F. The use of implants in the occlusal rehabilitation of a partially edentulous patient: a clinical report. J Prosthet Dent. 2001; 85:540-543.
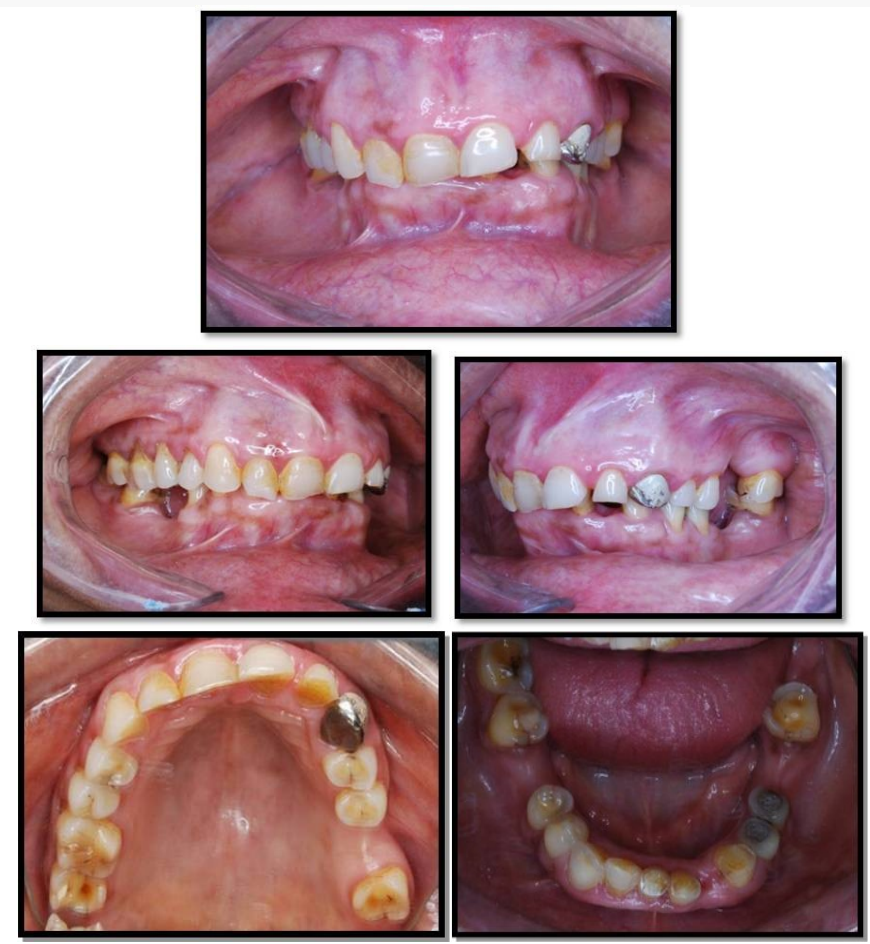

Pre-Operative Clinical Photograph 


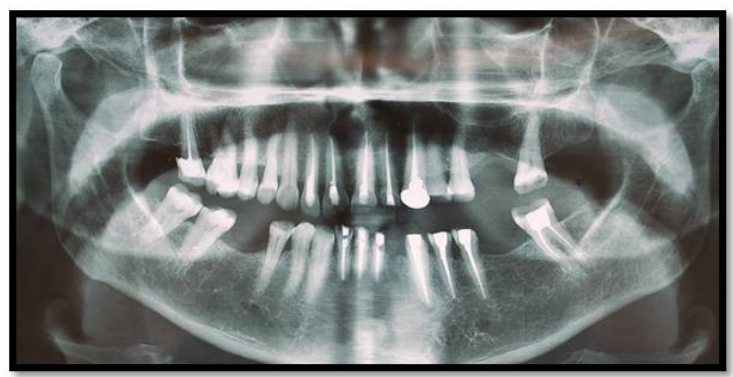

Diagnostic Opg

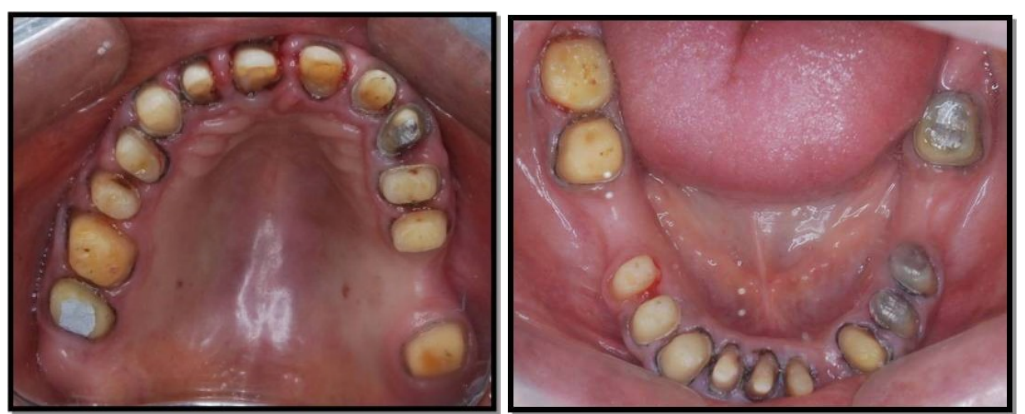

Intra Oral View Showing Tooth Preparation

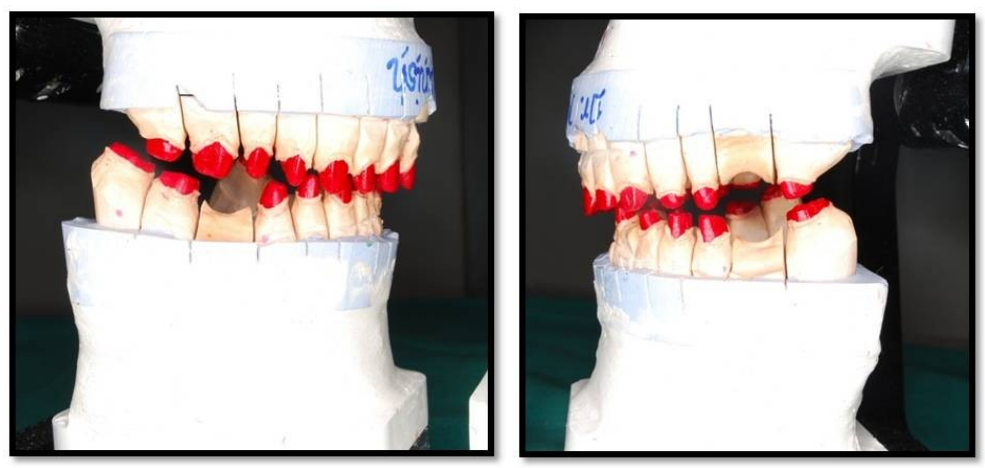

Wax Up On Working Model

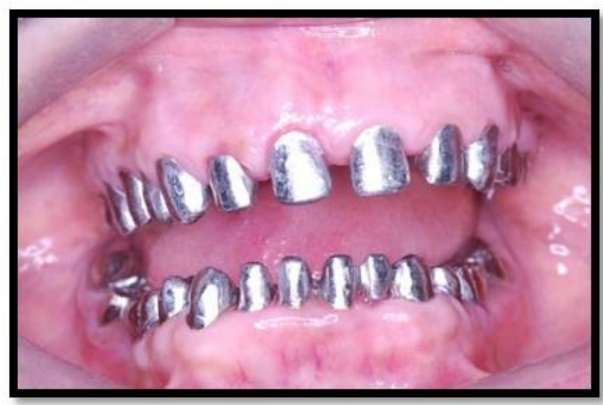

Metal Coping
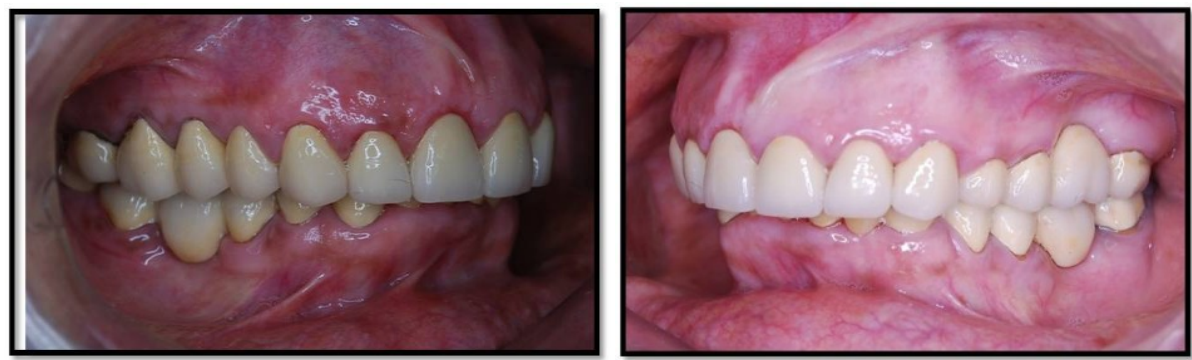

Intra Oral View after Crown Insertion 


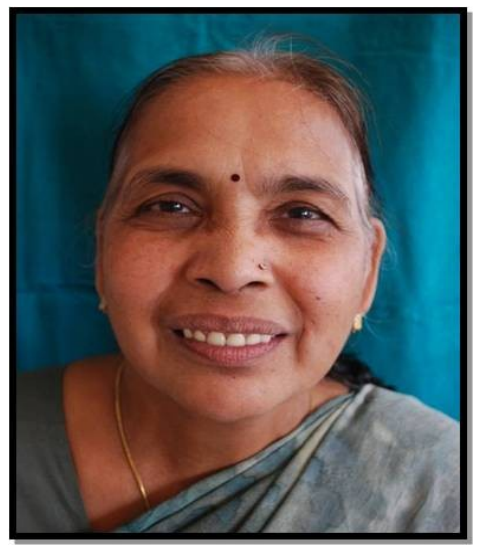

Post Operative

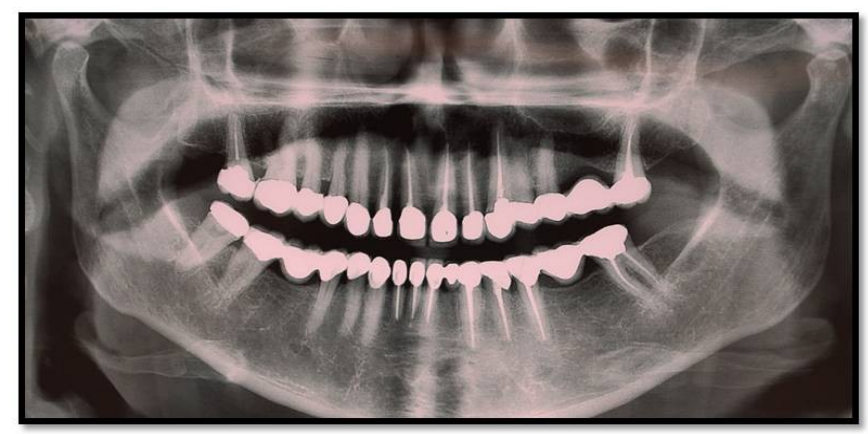

Post Operative Opg 